\title{
A multi-model approach to stakeholder engagement in complex environmental problems
}

\author{
E.A. Fulton ${ }^{\text {a }}$, T. Jones ${ }^{\text {b }}$, F. Boschetti ${ }^{a}$, M. Sporcic ${ }^{\text {a }}$, W. De LA Mare ${ }^{\text {a }}$, G. Syme ${ }^{\text {, }}$, P. Dzidic, \\ R. Gorton ${ }^{\text {, }, ~ L . R . ~ L i t t l e ~}{ }^{\text {, }}$, G. Dambacher ${ }^{a}$, and K. Chapman ${ }^{b}$ \\ ${ }^{a}$ CSIRO CMAR, Email: Fabio.Boschetti@csiro.au \\ ${ }^{b}$ Edith Cowan University, Western Australia
}

\begin{abstract}
We describe the different types of models we used as part of an effort to inform policy-making aiming at the management of the Ningaloo coast in the Gascoyne region, Western Australia. This provides an overview of how these models interact, the different roles they cover, how they fit into a full decision making process and what we learnt about the stakeholders involved in our project via their use.
\end{abstract}

When modelling is explicitly used to address socio-ecological issues, the key determinant of success is whether the models, their results and recommendations are taken up by stakeholders; such uptake in turn depends on addressing stakeholders' concerns, on engaging them in the project, on ensuring they feel ownership of the decision process at large, and that they understand and trust the modelling effort. This observation has guided our approach and has resulted in treating 'building a model' as the catalyst, rather than the final aim, of the process. In other words, extensive interactions in order to introduce, showcase, discuss and tune the model used for final decision making have represented both a requirement and an opportunity to ensure (i) model relevance, (ii) its acceptance, (iii) that all information available in the stakeholder team was accounted for and (iv) that stakeholders holding different levels of understanding of modelling, what it does and what it can provide to decision-making could develop an informed opinion on its use.

To fulfil these roles we developed five broad classes of models: conceptual models, toy-models, singlesystem models, shuttle-models and a full-system model. In conceptual models the main drivers of a system are highlighted for subsequent representation as components of the full-system model. This usually results in a diagram summarising our understanding of how the system works. In toy-models a problem is simplified in such a way that only a handful of components are included. The purpose of these models is mostly educational: we want to understand how each component affects the problem and in order to achieve this, we temporarily renounce a satisfactory understanding of the overall problem. In single-system models we include a fairly detailed representation of a single component of the system (in our case recreational fishing and tourism); these models can be used to introduce stakeholders to modelling, provide temporary results from the study of a single activity, which will feed into the development of the final full-system model, or address sector-specific issues. In shuttle-models, we include the minimum number of processes we believe are crucial for a basic understanding of the overall problem. We know these models are still too simple for full system description, but they provide a sufficient understanding to enable us to contemplate, build and use the more complex models needed for full problem description. The term 'shuttle' refers to taking us from a minimum to a full description of the problem, a journey which is necessary both to developers in model definition and parameterisation and to stakeholders in the interpretation of the final full-system model results. Finally, the full-system model includes all information collected through the project and addresses all scenarios of stakeholders concern, and whose definition has been greatly eased by use of the 'simpler' models.

As an example, a conceptual model may identify fishing and tourism as the main drivers of a region; a toymodel may describe how catches affect fish stocks; a single-system model may include the effect of gear, regulations and other processes affecting recreational fishing; a shuttle-model may include a simplified representation of the interaction between fishing, tourism, and infrastructure development on the overall health of the local ecosystem; this will gradually 'take' us to comprehend the 'full' model which may include tourism pressure, fish market values, climate effect, larger food-webs, etc.

Keywords: Modelling, Stakeholder engagement, Ecosystem management. 


\section{INTRODUCTION}

This work focuses on the role of multiple computer models in a large Management Strategy Evaluation project. Modelling is a crucial component of the approach, allowing for evaluation of the likely outcomes of available interventions before implementing them in the real world. There are other benefits modelling can provide, which relate to engaging different parties involved in a MSE project, including researchers, decision-makers and stakeholders. Here we focus on three specific items: 1) allowing a less biased interpretation of available information, 2) allowing for learning of specific skills and attitudes needed when facing complex problems and 3) providing an avenue for communication and collaboration.

Allowing a less biased interpretation of available information is important because people with different worldviews may interpret and draw very different conclusions from the same information. Research on attitudes to climate change, nanotechnology and vaccination, among other issues, show how worldviews affect policy support more than available information, because they filter how such information is processed. Accounting for such biases in a model (by parameterising the model according to different worldviews, (Boschetti 2011)) may be a way to highlight the issue and the potential inconsistencies which may arise from it, and to move the discussion from perspectives to mechanisms, in the hope that this may reduce the influence of biased information interpretation.

Training on specific skills and attitudes needed to face complex problems is important because scientific insights risk being lost unless they are understood by those making and supporting decisions. Recent studies have shown that human cognition and psychology affects decision-making at least as much as the complexity of the problem at hand (Moxnes 1998; Sterman 2008; Moxnes and Saysel 2009). Worse still, these difficulties are not necessarily obvious and may be confused as purposeful decision making (Moxnes 2000). Computer models, resembling flight simulators, can be designed to train individuals to better understand the basic processes of real world significance for decision making, including management of limited resources and unexpected feedbacks. The belief underneath this approach is that managing and predicting complex behaviours can be learned and that models can represent systems in a manner appropriate for learning and training.

Not only cognitive skills, but also cognitive attitudes are crucial to effective decision making in complex problems (Dorner 1996; Stanovich 1999; Sorrentino and Roney 2000). The behavioural attributes and habits we bring into a problem, the way we formulate goals, we interpret outcomes against expectations, we balance emotional responses like humility, curiosity, frustration and blame-shifting have a significant influence on how effectively we deal with complex situations (Dorner 1996). Tangible, constructive means to improve problem-solving in complex settings can be identified and trained via computer models (Dorner 1996; Boschetti, Hardy et al. 2010). Interestingly, some of the most effective cognitive approaches (including tolerating high levels of uncertainty, acknowledging mistakes, searching for counter evidence, self-reflection) can be in direct opposition to behaviours rewarded in political and management roles. More widespread awareness of what makes an effective decision maker, possibly leading to improvements in training programs, may have an immense impact on a wide variety of real world issues.

Finally, the key determinant of success in MSE lies on whether the models, their results and their recommendations are taken up by stakeholders. Evidence suggests that such uptake in turn depends on addressing stakeholders' concerns as well as on engaging them in the project. Extensive interactions in order to introduce, showcase, discuss and tune the models represent an opportunity to ensure that the models are relevant to businesses, managers and communities, have sufficient trust to be considered for ongoing use, receive political legitimacy and provide improved coordination for making decisions, which are the required characteristics for a successful collaborative planning process (Bramwell and Sharman 1999; Aas, Ladkin et al. 2005).

A single model can rarely fulfil so many diverse tasks. A full-system model used to predict the impact of policies accounting for feedback cycles at different scales is unlikely to represent the best tool to train users to understand the effect of simple stock and flows processes. Similarly, the latter is unlikely to be effective to convince stakeholders that models can help their community and the development time involved in large models means there is a significant risk of a loss of interest or knowledge of the project (due in part to a turnover in the stakeholders in regulatory bodies and the local population). Here we describe a suite of models we have developed to fulfil the different roles for models within a MSE project. Each model will be briefly described; most important, we will discuss how the models have been used at different stages in the project and the main lessons learned. 


\section{WHY WE MODEL}

Modellers themselves often have very different ideas of what models do and what their output represent (Boschetti, McDonald et al. 2008; Boschetti, Grigg et al. 2011). For example, models may be used to: 1) predict the behaviour of a system, 2) retrodict (understand what led to an observed situation), 3) understand how a system functions, 4) understand possible causal relations, 5) explore system behaviour, 6) build tools for others to use, 7) teach how a system functions, 8) train specific skills and develop useful learning attitudes, 9) communicate formalised knowledge, 10) foster communication and collaboration. All these items have a role at different stages of a successful MSE. Here we focus on a challenge which a modelling team is likely to face: while for natural scientists using a model to synthesise system understanding and predict its behaviour is an obvious procedure (is what science is about), for many stakeholders this is a fairly esoteric, speculative, abstract and unrealistic way to address a problem. This may result in scepticism which may prevent communication and trust. Effort needs to be invested in overcoming this and the following two approaches may be useful. First, it is important to realise that modelling and predicting is what we all do whenever we plan and implement a daily action; this modelling however happens in our brain, not in a computer. Toy-models (described below) can be useful to show how the two processes (mental and formal modelling) relate and how a computer model can be useful to overcome the mistakes which may occur when dealing with complex or unfamiliar problems. Second, it is likely that all stakeholders are already familiar with the concept of modelling but not aware of it: we are all in general recipients of model results, for example by interpreting and making decisions on weather bulletins and stock market projections. Showing the analogies between different types of models and involving stakeholders in the process of conceptual model building, followed by regularly showcasing model development and temporary results, can accustom stakeholders to the types of models used in MSE. This continuous work is essential for stakeholder to trust, understand and take ownership of the final model results.

\section{TYPES OF MODELS}

We group the models we have used into five broad classes: conceptual models, toy-models, single-system models, shuttle-models and full-system models, as describe in the Extended Abstract. Here we describe these types of models, (with the exception of conceptual models) and how they have been used in the Ningaloo Research Program. The relation between the different purposes for using models in a MSE and types of models, is summarised in Table 1.

Table 1. Relation between modelling purpose and model types

\subsection{Conceptual models}

Conceptual models contribute to model development rather than providing a usable model of themselves. There is some confusion around the term as it can apply broadly to any model that an individual uses to explain an outcome, or more narrowly to a type of modelling that does not use quantitative data. Here, we use the

\begin{tabular}{|l|l|l|l|l|l|}
\hline Purpose/Type & Concept. & Toy & Single & Shuttle & Full \\
\hline Predict/Retrodict & & & $\bullet$ & & $\bullet$ \\
\hline Understand system functioning & $\bullet$ & $\bullet$ & & $\bullet$ & $\bullet$ \\
\hline Understand causal relations & $\bullet$ & $\bullet$ & $\bullet$ & $\bullet$ & $\bullet$ \\
\hline Explore system behaviour & & & $\bullet$ & $\bullet$ & $\bullet$ \\
\hline Build tools for others to use & & $\bullet$ & $\bullet$ & $\bullet$ & $\bullet$ \\
\hline $\begin{array}{l}\text { Train specific skills and develop } \\
\text { useful learning attitudes }\end{array}$ & $\bullet$ & & & $\bullet$ & \\
\hline $\begin{array}{l}\text { Foster communication and } \\
\text { collaboration }\end{array}$ & & & $\bullet$ & $\bullet$ & $\bullet$ \\
\hline
\end{tabular}
term conceptual modelling to refer to conceptual diagrams that summarise relationships within a system or between systems, but do not contain quantitative data. As such, conceptual models were used as part of the development of the single-system, shuttle and full-system models in particular.

\subsection{Toy-models}

Two types of toy-models have been developed mostly for educational purposes: stocks \& flows and feedback loop models. The stocks \& flows model (Boschetti, Fulton et al. 2010; Boschetti, Hardy et al. 2010) allows us for interactive control of the in and out flows into a bathtub, as inspired by (Sweeney and Sterman 2000)). A user is asked to predict the amount of water in the bathtub as a function of how much water enters and exits at different times. As explained in (Sweeney and Sterman 2000), even well educated people often fail at predicting the behaviour of this apparently simple process. Since all systems depend on a careful balance of resource usage (water, energy, people, $\mathrm{CO}_{2}$, biological species) understanding these process is crucial for effective management. 
The feedback loop model (Casagrandi and Rinaldi 2002; Boschetti, Fulton et al. 2010; Boschetti 2011) allows us to study the interaction between three abstract variables: 1) the size of a population exploiting a resource, 2) the way exploitation is carried out and 3) the dynamics of the environment which provides the resource. By specifying the nature of the population and type of resource, this abstract representation can be applied to a range of different problems, including fishery management, water conservation, tourism development and climate change. This type of model can help understand the medium and long-term effect of positive and negative feedback loops, develop an intuition for the role and impact of specific links on system behaviour and where points of intervention may lie.

\subsection{Single-system models}

Two single-system models have been used: a fishery model (ELFSim) and a tourism destination model. ELFSim (Little, Punt et al. 2007) is a fishery decision support tool designed to evaluate options for conservation and harvest management. It includes three conceptual modules: a) a biological module, which can model both sedentary and mobile target species and incorporates several features of the species of interest, including larval settlement/transport, age-related structure and sub-populations; b) an effort allocation module, which projects historical fishing data into the future and c) a management module, which evaluates the likely outcomes of different management options, including area closures, minimum catch size and gear selection, in terms of biological and economic performance indicators. An ELFSim simulation consists of two parts. In the first, the biological module uses information from the physical characteristics of individual reefs to determine the population size and structure on each reef given past fishing. The second part projects the reef populations forward in time according to simulated fishing pressure and provides biological and economic performance indicators thus allowing evaluation of alternative management options.

The tourist destination model assesses the economic, social and environmental impacts of different planning decisions, broader contextual shifts (such as a resource boom or terrorism event), changes in tourism numbers and tourism segments and specific events (Jones, Glasson et al. 2011). It aims to explore alternative futures related to infrastructure development, tourism growth, external economic impacts, resource use, perception of a location natural attractiveness, service delivery, energy consumption, waste generation and specific system shocks such as cyclones, pandemics and loss of a significant natural assets (for example coral bleaching and changing patterns of iconic wildlife visitation).

\subsection{Shuttle-models}

Two shuttle-models were also used in this project: ScenarioLab, focussed mostly on fishing and Ecopath with Ecosim (EwE), which includes a larger system view. ScenarioLab (Boschetti, de La Tour et al. 2008; Boschetti, Fulton et al. 2010) allows for rapid assessment of the effect of decision-making on fishery management by allowing a team of non-expert modellers to compare the outcomes of multiple model simulations via a set of GUIs. A distinguishing feature is that the goal of the management strategies can arise as a result of the interaction between the user and the model, rather than being defined a priori, and can change during the process in response to the accumulated information and insights. ScenarioLab estimates the expected effect of a number of fishery regulation options available to the management team, which control the number of dispensed fishing licences, the total areas reserved as sanctuary zones, daily catch limits, and minimum and maximum legal lengths for two species of interest. Once these regulations are defined, the model mimics the behaviour of a fishing fleet and its effect on the modelled foodwebs. For each regulation option, the model is run a number of times under different model settings, in order to mimic natural and parameterisation uncertainty.

The Ecopath with Ecosim (EwE) (Christensen 2011) modelling approach was originally developed to explore the consequences of fishing, environmental disturbances and human behaviour on the dynamics of marine foodwebs. The approach and software has been under development for over 25 years and combines ecosystem trophic mass balance analysis (Ecopath, released in 1984), with a temporally dynamic modelling capability (Ecosim, released in 1995) for exploring past and future impacts of exploitation and environmental disturbances. In 1998 Ecospace was added to the package, this replicates Ecosim models over a spatial map grid to allow exploration of policies such as marine protected areas, while accounting for spatial dispersal/advection effects. Together the three components form an integrated package that is relatively straightforward to use and has been applied to over 200 locations around the world. Ningaloo-EwE contains 53 ecological groups, spanning both terrestrial and marine components, ranging from primary producers to top predators. EwE was originally written to consider the effects of fisheries but it is possible to include a 
broader set of human activities, including agriculture, shipping, camping and tourism, making EwE a perfect shuttle-model candidate.

\subsection{Full-system model}

The InVitro modelling framework (Gray, Fulton et al. 2006) has been used to study the interrelation among all processes of interest in the full Ningaloo system. It allows for modelling of processes across scales and components. As it brings together all main system components (biophysical, ecological, socio-economic), it helps highlight tradeoffs between the demands of different economic activities and the requirements for social and ecological sustainability. Importantly, it captures feedback processes that can lead to unexpected changes in the system. The cross-scale capability is achieved using a hybrid framework that combines analytical, equation-based formulations for physical or lower trophic level processes with algorithmic, rulebased formulations for the higher trophic levels and humans, each framework acting at the appropriate time and spatial scale. InVitro consists of 5 main sub-models: 1) biophysics, which defines the natural environment, 2) socio-economics, which defines human behaviour, 3) industry, which defines large-scale economic drivers and institutions, 4) management, involving the decision making and 5) monitoring and assessment. This modular design provides flexibility by choosing alternative representations of the each submodel depending on the specific modelling task as well as a means to handle uncertainty by jointly testing and implementing multiple representations of the same module.

The biophysical sub-model includes all the dominant system components of a 60 species foodweb (primary producers, benthic habitats, benthic invertebrate communities, pelagic forage fish, main target species of finfish and crustaceans, top predators and species of special interest and detailed representation of several physical processes (oceanography, weather, climate, geomorphology, contaminants, etc). The industry submodel includes commercial and recreational fisheries, tourism, oil and gas, salt production, coastal development and infrastructure, ports, shipping, regional economics, catchment use, recreation and conservation. The effects of human activities on the marine environment are represented using a combination of analytical decision models, response functions, specified rules, historical data and scenarios. Uncertainty is included in each of these options and determines which exact activity is carried out and its outcome; this aims to capture natural ambiguity in human and animal behaviour, missing or incorrect information and

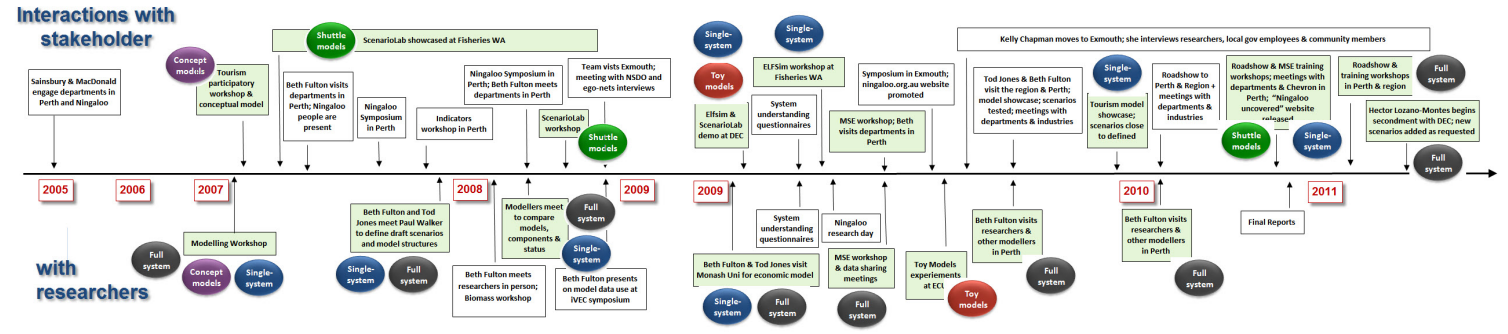

Figure 1. Different model types during the project (A larger version is available at http://www.per.marine.csiro.au/staff/Fabio.Boschetti/images/Ning1.jpg)

unpredictable events.

\section{MODEL USE AND OUTCOMES}

Figure 1 summarises when and within what context the different models types have been used in relation to the stakeholder engagement activities. The relative ad-hoc nature of the models development, which followed opportunities and needs as they arose rather than an organic plan, is noticeable from the sparse formal use of conceptual models and the use of toy-models fairly late in the project, rather than at the beginning, when most training is likely needed. It is also likely that shuttle models have been under-utilised. This has been a learning experience for the modelling team, as is discussed in the next session.

This project's outcomes can be divided into four broad classes, each significant to a different type of audience: a) model results, b) actual models, c) stakeholder learning and education in model use and d) learning for the modellers themselves. 
The output from the full-system model (InVitro) and the two single-system models (Ningaloo Tourism Destination model and ELFSim) will be interpreted in collaboration between researchers and decision makers and will feed both into the decision making and consultation processes. Some of these results will also be available to the general public via a number of web sites. While we do not expect that all stakeholders will want to actively use the models, feedback from purposely designed questionnaires and casual conversation suggest that the stakeholders who got involved more closely with the modellers, via presentations and workshops, have acquired a deeper understanding of what models can provide, and how they can be used in decision making. This is promising; to what extent this promising result is due to the selected feedback we received, and to what extent such learning has been achieved in the overall stakeholder group is something which is worth monitoring in, say, 6-12 months from project completion. This information can feed into better future training and interaction processes.

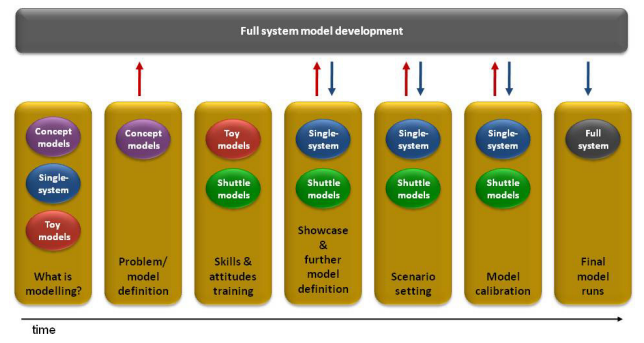

Figure 2. Stakeholders engagement phases and use of different model types.

Finally, considerable learning has occurred within the modelling group. This relates not only to the engagement process and to modelling itself, which always improves with accumulated experience and technology, but specifically to how to employ different types of models within different engagement phases. Figure 2 summarises this learning visually; it includes 7 stages going from the first engagement and model building activity to the final delivery and discussion of the modelling results and for each it shows what types of models that can be useful when tackling such a large and complex problem. Importantly, it also includes the relation to the software-engineering activity of building the full-system model which requires an organic project plan from project inception. While the exact details of which toy or shuttle model are needed may develop during the project, and a certain level of flexibility will be required, maintaining a relation between these models and the full-system model is beneficial. For example, a common user interface may allow users to accustom themselves to the different models without re-training and can provide more specific information of the GUI effectiveness for the final model.

\section{DISCUSSION}

Traditionally, MSE includes stakeholder engagement to define project goals, plans, monitoring and learning, while it employs a model to assess the likely outcomes and trade-off from possible alternative plans. As described in Figure 3, our MSE implementation differs in three important features. First, modelling has been used at each stage of the process, to engage stakeholders, facilitate learning, elicit
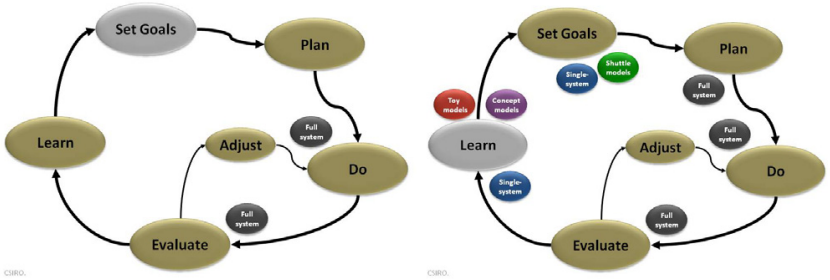

Figure 3. Traditional MSE implementation (left); proposed MSE implementation, in which different types of models are used at each stage of the process (right). (A larger version is available at http://www.per.marine.csiro.au/staff/ Fabio.Boschetti/images/Ning2.jpg) feedback and improve model design. While the Full Model was not available for use until the very end of a long project, the other models provided opportunities for learning and engagement throughout the modelling process. Hence the learning and goal setting was supported by modelling throughout development, with regular presentations to key organisations and stakeholder meetings. Such engagement encouraged exploration of issues through use of the single system and shuttle models, often evoking further questions that contributed to development of the full model. An example was a request to examine a fishing strategy with strong local support. Wilderness fishing involves anglers only keeping enough fish for them to eat that evening. This management strategy was evoked through communications for a model demonstration, was able to be explored by stakeholders as part of the process of model development.

Second, different types of models have been used for different purposes and for different audiences, including conceptual models to capture system functioning, toy-models for training, single-component models to highlight and understand specific drivers in the system, shuttle-models to introduce system complexity and full-system model to deliver the final data. 
Fulton et al., A multi-model approach to stakeholder engagement

Finally, these models have allowed for the MSE process to be implemented differently. While traditionally MSE begins by identifying goals (as on the left hand side of Figure 3), models can be used to start the MSE process with learning (as on the right hand side of Figure 3). This step can be particularly useful in future projects since some stakeholders groups in our project found it difficult to formulate MSE questions because of their lack of familiarity with modelling; overcoming this difficulty with a suitable training in the philosophy and use of models can considerably speed up the overall process at later stages. Furthermore, modelling-facilitated learning can occur throughout the process, rather than at the end of model development. While group-learning should occur regardless of the presence of usable models, using toy, single-system and shuttle models adds another dimension to the learning that enables greater engagement with the problems of managing complex and non-linear systems.

\section{REFERENCES}

Aas, C., A. Ladkin, et al. (2005). "Stakeholder collaboration and heritage management." Annals of Tourism Research 32(1): 28-48.

Boschetti, F. (2011). "A simple model of how people reason about climate change " In preparation.

Boschetti, F., A. de La Tour, et al. (2008). "Interactive modelling for natural resource management Submitted.

Boschetti, F., E. Fulton, et al. (2010). Simple computer models in the management of complex ecological systems. N. C. C. F. Report. Perth.

Boschetti, F., N. J. Grigg, et al. (2011). "Modelling = conditional prediction." Ecological Complexity 8(1): 86-91.

Boschetti, F., P. Hardy, et al. (2010). "Can we learn how complex systems work?" Emergence: Complexity and Organization In Print.

Boschetti, F., D. McDonald, et al. (2008). "Complexity of a modelling exercise: a discussion of the role of computer simulation in Complex System Science." Complexity 13(6): 21-28.

Bramwell, B. and A. Sharman (1999). "Collaboration in local tourism policymaking." Annals of Tourism Research 26(2): 392-415.

Casagrandi, R. and S. Rinaldi (2002). "A theoretical approach to tourism sustainability." Ecology and Society 61(1): 13.

Christensen, V. (2011). Ecopath with Ecosim: Linking fisheries and ecology. . Handbook of Ecological Modelling, Network and Informatics. . S. E. Jørgensen, CRC Press.

Dorner, D. (1996). The Logic Of Failure: Recognizing And Avoiding Error In Complex Situations. Ney York, Metropolitan Books.

Gray, R., E. Fulton, et al. (2006). Ecosystem model specification within an agent based framework. Hobart, Tasmania, CSIRO Australia.

Jones, T., J. Glasson, et al. (2011). "Regional Planning and Resilient Futures: Destination Modelling and Tourism Development - the case of the Ningaloo Coastal Region in Western Australia." Planning Practice and Research 26(4): 393-415.

Little, L. R., A. E. Punt, et al. (2007). "ELFSim--A model for evaluating management options for spatially structured reef fish populations: An illustration of the "larval subsidy" effect." Ecological Modelling 205(3-4): 381-396.

Moxnes, E. (1998). "Overexploitation of renewable resources: the role of misperceptions." Journal of Economic Behavior and Organization 37(1): 107-127.

Moxnes, E. (2000). "Not only the tragedy of the commons: misperceptions of feedback and policies for sustainable development." System Dynamics Review 16(4): 325-348.

Moxnes, E. and A. K. Saysel (2009). "Misperceptions of global climate change: information policies." Climatic Change 93: 15-37.

Sorrentino, R. and C. Roney (2000). Uncertain Mind: Individual Differences in Facing the Unknown Philadelphia, Psychology Press.

Stanovich, K. (1999). Who is rational? : studies of individual differences in reasoning Lawrence Erlbaum Associates.

Sterman, J. D. (2008). "Risk Communication on Climate: Mental Models and Mass Balance." $\underline{\text { Science }}$ 322(5901): 532-533.

Sweeney, L. B. and J. D. Sterman (2000). "Bathtub dynamics: initial results of a systems thinking inventory." System Dynamics Review 16(4): 249-286. 